\title{
Utilización de servicios de salud por enfermedades catastróficas o de alto costo en Antioquia*
}

\section{Use of Health Services for Catastrophic or High-Cost IIInesses in Antioquia}

\section{Utilização de serviços de saúde por doenças catastróficas ou de alto custo em Antioquia}

Fecha de recepción: 20-04-2016 Fecha de aceptación: 30-03-2017 Disponible en línea: 30-05-2017 doi:10.11144/Javeriana.rgps16-32.usse

Cómo citar este artículo:

Lopera-Medina MM. Utilización de servicios de salud por enfermedades catastróficas o de alto costo en Antioquia. Rev Gerenc Polít Salud. 2017; 16 (32): 120-137. https://doi.org/10.11144/Javeriana.rgps16-32.usse

Artículo de investigación. Este trabajo es uno de los productos derivados de la investigación Caracterización clínica, sociodemográfica y financiera de los eventos de alto costo en Antioquia, 2012-2013, financiada por el Fondo de Apoyo Docente de la Facultad Nacional de Pública, Universidad de Antioquia y la Estrategia de Sostenibilidad de Comité para el Desarrollo de la Investigación (CODI) de la Universidad de Antioquia 2012-2013. La investigación se ejecutó entre el 15 de junio del 2014 y el 20 de junio del 2016.

** Ph. D. en Salud Pública, bacterióloga y laboratorista clínica, profesora de la Facultad Nacional de Salud Pública de la Universidad de Antioquia. Dirección de correspondencia: Calle 62 N.o 52-59, Oficina 306. Correo electrónico: monica.lopera@udea.edu.co 


\section{Resumen}

Las enfermedades catastróficas o de alto costo (EAC) causan alta morbimortalidad y demandan atención permanente, compleja y costosa. Objetivo: caracterizar la utilización de servicios de salud por enfermedades de alto costo. Método: estudio descriptivo transversal. Se analizó información de los registros individuales de prestación de servicios para ocho EAC, usando codificación CIE-10. La utilización se analizó según variables sociodemográficas y características de la oferta. Resultados: el $16 \%$ de la utilización de servicios fue atribuible a pacientes con EAC. Esta fue más frecuente en pacientes con enfermedad renal crónica y precursoras (ERC-P), enfermedades huérfanas y epilepsia, mujeres mayores de 50 años y personas del régimen contributivo (con variaciones según la enfermedad). Entre 66 y $83 \%$ de la utilización se produjo en instituciones privadas y predominó la atención por medicina general, con escasa participación de otras áreas de salud. Conclusión: se encontraron diferencias inaceptables de utilización entre regímenes de afiliación y variables de oferta, afines a las políticas privatizadoras.

Palabras clave: utilización; enfermedad catastrófica; atención ambulatoria; hospitalización; servicios médicos de urgencia; personal de salud

\section{Abstract}

Catastrophic or high-cost illnesses (HCI) cause high morbidity and mortality, and demand permanent, complex, and expensive care. Objective: to characterize the use of health services due to high cost illnesses. Method: cross-sectional descriptive study. We analyzed information from individual service rendering records for eight HCIs, using CIE-10 coding. The use was analyzed according to sociodemographic variables and characteristics of the offer. Results: $16 \%$ of the service use was attributable to patients with HCI. This was more frequent in patients with chronic renal disease and precursors (ERC-P), orphan diseases, and epilepsy; women over 50 years of age and people in the contributory regime (with variations depending on the disease). Between 66 and 83 percent of the use occurred in private institutions and care through general medicine was prevailing, with little participation of other health areas. Conclusion: unacceptable differences of use were found between affiliation regimes and supply variables, related to privatization policies.

Keywords: healthcare utilization; catastrophic illness; hospitalization; ambulatory care; emergency medical services; health personnel

\section{Resumo}

As doenças catastróficas ou de alto custo (EAC) ocasionam alta morbimortalidade e demandam atenção permanente, complexa e custosa. Objetivo: caracterizar a utilização de serviços de saúde por doenças de alto custo. Método: estudo descritivo transversal. Analizou-se informação dos registros individuais de prestação de serviços para oito EAC, usando codificação CIE-10. A utilização analizou-se segundo variáveis sociodemográficas e características da oferta. Resultados: o $16 \%$ da utilização de serviços foi atribuível a pacientes com EAC. Esta foi mais frequente em pacientes com doença renal crónica e precursoras (ERC-P), doenças orfanas e epilepsia, mulheres maiores de 50 anos e pessoas do régime contributivo (com variações segundo a doença). Entre 66 e $83 \%$ da utilização produziu-se em instituições privadas e predominou o atendimento por medicina geral, com escassa participação de outras áreas da saúde. Conclusão: verificaram-se diferenças inaceitáveis de utilização entre regímenes de afiliação e variáveis de oferta, afins às políticas privatizadoras.

Palabras clave: utilización; enfermedad catastrófica; atención ambulatoria; hospitalización; servicios médicos de urgencia; personal de salud 


\section{Introducción}

La comprensión de los perfiles de utilización de servicios de salud en los casos de enfermedades de alto costo es una pieza clave en la planificación de los sistemas de salud. En conjunto con otros datos de necesidad, prevalencia e incidencia, permite modelar la disponibilidad, la oferta y las redes de los servicios de atención requeridos para atender las condiciones particulares de los territorios, una vez analizadas las necesidades en salud para cada una de las enfermedades.

Los perfiles de utilización constituyen indicadores de desempeño de los sistemas de salud, por cuanto no solo muestran los procesos llevados a cabo en la organización de los servicios, sino que también permiten evidenciar resultados en términos de eficacia y equidad. La comprensión de estos fenómenos tiene el potencial, en un marco ético de la política pública, de determinar las condiciones financieras necesarias para la operación del sistema de salud y, por lo tanto, mejorar la eficiencia en la asignación de recursos.

Si bien la utilización es uno de los indicadores de gestión de los servicios, no es un indicativo completo de las necesidades en salud, puesto que en ella median múltiples procesos, relacionados con aspectos de los individuos y sus familias, pero también con factores asociados a la organización y oferta sanitaria. En cuanto a los factores del individuo, la utilización se asocia con la necesidad, el deseo y la búsqueda. La necesidad puede derivarse de la autopercepción de la alteración de salud o bienestar, los síntomas, el tipo de enfermedad, el tiempo de incapacidad, así como de la percepción de restricción biológica o social para el desarrollo de las actividades cotidianas $(1,2)$. El deseo de atención depende de las creencias relativas a la salud, la tolerancia al disconfort y la confianza en el sistema de atención médica (2).
Con relación a las características de oferta de los servicios de salud, los factores asociados con la utilización, tanto de prevención como de atención sanitaria, están asociados a la disponibilidad, el seguro de salud, la relación con el personal asistencial, la accesibilidad temporal (referida a los tiempos de operación del servicio), la distancia de los servicios, los costos y el nivel de satisfacción con los servicios $(1,2)$.

La utilización tampoco es un indicador de acceso a los servicios de salud. Es posible que exista necesidad pero no se utilicen los servicios. Ya algunos autores han descrito que existe una proporción de personas, que oscila entre el 60 y el $70 \%$, que requieren y no acceden a este. Tal fenómeno ha sido descrito por Last desde la década de los sesenta como el "iceberg de la enfermedad" (1). Por el contrario, también puede existir un patrón de hiperutilización, injustificado desde el punto de vista médico, relacionado con la condición clínica, la frecuencia, la intensidad o la sofisticación del servicio que no compensa el beneficio. Esta hiperutilización se asienta en múltiples causas (3-5).

A pesar de las anteriores limitaciones, el estudio de la utilización sí permite entender la forma como se concreta la atención de los pacientes con ciertas enfermedades, es decir, las características resultantes de la confluencia entre la búsqueda de servicios y la respuesta por parte de la oferta. A escala nacional, se sabe que las enfermedades no transmisibles absorben el $65 \%$ de la atención en salud (6). Este grupo, sin embargo, no contempla otras enfermedades reconocidas como de alto costo en el país, de las cuales se carece de información.

La utilización de los servicios de salud por las poblaciones que padecen enfermedades de alto costo (EAC o catastróficas) pudiera exhibir un perfil diferencial, toda vez que 
estas enfermedades son complicadas desde el punto de vista clínico. La mayoría son crónicas y progresivas, pueden ser discapacitantes o dejar secuelas residuales, en especial en ausencia de programas de control eficaces. Lo paradójico es que muchas de estas enfermedades son prevenibles, pero cuando se presentan demandan atención prolongada, continua, costosa y de alta complejidad.

La normatividad colombiana define las EAC como aquellas que representan una alta complejidad técnica en su manejo, alto costo, baja ocurrencia y bajo costo-efectividad en su tratamiento y en la modificación del pronóstico (7). Hasta el momento, se ha producido una lista de ocho EAC que son reportadas por las empresas administradoras de planes de beneficio (EAPB) a la cuenta de alto costo(CAC), pero no se cuenta con un listado completo y exhaustivo de las enfermedades por categorías y subcategorías, ni tampoco de los tratamientos considerados de alto costo. En este grupo de enfermedades están incluidas enfermedades no transmisibles como el cáncer, la enfermedad cardiovascular, la enfermedad renal crónica (ERC) y sus precursoras - diabetes e hipertensión arterial (HTA)—, las enfermedades huérfanas, que en Colombia incluyen aquellas raras, ultrahuérfanas y olvidadas. Bajo esta denominación, las enfermedades huérfanas constituían para la fecha una lista de 1947 enfermedades. De ellas, aproximadamente diez mil afectan también órganos del sistema cardiovascular o renal. Estas enfermedades son consideradas crónicas, debilitantes y graves, dado que amenazan la vida de quienes la padecen $(8,9)$. Otra de las enfermedades de alto costo es el VIH/ sida, una enfermedad crónica transmisible. Entre las enfermedades que afectan otros órganos y sistemas se consideran de alto costo aquellos problemas neurológicos como la epilepsia, enfermedades reumá- ticas, procesos relacionados con el trauma mayor que requiere atención de cuidados intensivos, otras enfermedades o problemas indefinidos que requieren cuidados intensivos, tratamientos quirúrgicos y trasplantes.

No obstante la definición anterior, basada en este grupo de ocho enfermedades, el Ministerio de Salud y Protección Social y otros entes encargados definieron como eventos catastróficos algunas enfermedades priorizadas "en razón de la carga financiera y las condiciones epidemiológicas" y determinaron los estadios de las enfermedades que serían reconocidos como eventos de alto costo, en virtud del procedimiento tratamiento "de alto costo" necesario (PTAC). Así, solo se consideran eventos de alto costo la enfermedad renal crónica en estadío 5 (ERC-5), que implica procedimientos PTAC como la diálisis y trasplante renal, la enfermedad cardiovascular (ECV), que amerita tratamientos quirúrgicos o trasplante de corazón, otras enfermedades que requieren trasplantes (médula ósea y córnea), algunos tipos de cáncer que implican tratamientos de radioterapia y quimioterapia, los tratamientos antirretrovirales y el manejo de complicaciones para el sida, reemplazos articulares, tratamientos quirúrgicos para enfermedades del sistema nervioso central, enfermedades genéticas o congénitas, el trauma mayor y la terapia en unidad de cuidados intensivos (7). La norma clasifica la atención por consulta externa, hospitalización y urgencias como de alto costo, si es compatible con los eventos o tratamientos definidos normativamente, y como consultas por enfermedad general cuando un paciente con EAC utiliza los servicios por causas que pueden o no estar asociadas con la enfermedad de base.

En el sistema de salud colombiano, la priorización de las enfermedades sugiere un desconocimiento de la integralidad requerida 
para la prestación de servicios, pero también desestima la complejidad clínica y la progresividad de la mayoría de estas enfermedades. En este sentido, se establece una dualidad artificial entre paciente con EAC, evento de EAC y PTAC, y, por lo tanto, desalienta la oportunidad en el diagnóstico, la integralidad de los tratamientos, la continuidad del manejo e incluso la utilización, puesto que impone barreras financieras relacionadas con copagos y cuotas moderadoras para las EAC no priorizadas.

\section{Epidemiología de las enfermedades catastróficas o de alto costo, un panorama mundial y local}

A pesar de que la norma colombiana considera la baja ocurrencia como uno de los criterios para la definición de EAC, estas enfermedades constituyen un problema creciente de salud, no solo en Colombia, sino en el mundo (10). En el mundo, la incidencia, la prevalencia y la mortalidad relacionadas con EAC van en aumento. Las enfermedades no transmisibles y precursoras constituyen la primera causa de muerte, tanto en países de bajos ingresos como en países de altos ingresos (11), y Colombia no es la excepción.

En el país, según el informe del análisis de Situación de Salud (ASIS) del 2015, la Cuenta de Alto Costo - (CAC) aún no tiene sistematizada la información correspondiente a la situación de salud para el grupo de las EAC, y solo registró información para ERC y VIH (6), pero otras fuentes permiten entender la situación epidemiológica de estas enfermedades. Entre el 2005 y el 2013, la principal causa de muerte en la población general fueron las enfermedades del sistema circulatorio. Estas han sido responsables del $29.9 \%$ de las defunciones y del $16.13 \%$

124 de todos los años de vida potencialmente perdidos (AVPP). Las neoplasias aportaron un $17.8 \%$ de las muertes y el $14.5 \%$ de los AVPP (6). De acuerdo con el informe de la CAC, en el año 2015, en el país existían 3385457 personas con hipertensión arterial, diabetes mellitus y ERC (12). La prevalencia de la ERC-5 fue de 72.9/100000 y la incidencia fue de 12.3 por 100000 afiliados. La prevalencia de hipertensión arterial fue de $6.5 \%$ (12), mientras que la prevalencia de diabetes fue de $1.9 \%$ (12). En cuanto al VIH, desde 1985 hasta el 31 de diciembre del 2013 se notificaron 92379 casos de VIH/ sida (6). En el 2013 la prevalencia estimada fue de 17.4 casos de VIH/sida por 100000 habitantes. En el 2011 se calculó una carga de enfermedad por VIH de 1013444 por AVPP (13).

En el país existen muy pocos estudios que den cuenta de la magnitud del problema de la epilepsia y de las enfermedades huérfanas. El estudio nacional Epineuro, realizado entre el 2003 y el 2006, encontró una prevalencia de epilepsia de 10.1-10.3/1000 habitantes $(14,15)$. Sobre las enfermedades huérfanas, se sabe que en el 2012 existían 13168 personas diagnosticadas (16).

En el departamento de Antioquia no existen registros integrados que den cuenta de la situación de salud por EAC, ni tampoco de los patrones de utilización de servicios para el manejo y control de estas enfermedades; sin embargo, existen algunos informes de la morbimortalidad que indicarían también una tendencia al aumento $(17,18)$. Por lo tanto, este estudio describe la frecuencia de utilización de los servicios de consulta externa, hospitalización y urgencias para cada EAC y su relación con algunas variables sociodemográficas y de la organización de la oferta. A efectos de dicho trabajo y de conformidad con una postura que aboga por la integralidad en la atención, los patrones de utilización hacen referencia a la utilización de servicios para resolver 
las necesidades en salud de todas las EAC definidas en Colombia, y no solo aquellas priorizadas o las que ameritan los PTAC explícitamente reconocidos.

\section{Métodos}

Tipo del estudio: se realizó un estudio observacional, de corte y retrospectivo.

Fuentes de información: los datos fueron extraídos de la base los registros de atención individual (RIPS) de Antioquia en el 2011. Las EAC seleccionadas fueron aquellas definidas en la Resolución 5261 de 1994: enfermedad renal crónica y precursoras (en adelante ERC-P), enfermedad cardiovascular (ECV), cáncer, enfermedades huérfanas, enfermedades neurológicas como epilepsia, trauma mayor (gran quemado), artritis y VIH. No se incluyeron enfermedades o problemas indefinidos como uso de cuidados intensivos o enfermedades indefinidas que ameritan cirugía o trasplante. Las enfermedades y sus subcategorías fueron seleccionadas de acuerdo con la clasificación de enfermedades CIE-10. La ERC-P incluyó los grupos establecidos por la National Kidney Foundation (19), es decir, la ERC en estadios 1-5 (ERC1-ERC-5) más la ERC en estado terminal. Entre las enfermedades precursoras se contemplaron la hipertensión (HTA) y la diabetes. La enfermedad cardiovascular (ECV) incluyó en cuatro categorías: infarto agudo de miocardio y enfermedades cardíacas isquémicas, cardiopatías reumáticas crónicas, enfermedad cerebrovascular y otras formas de cardiopatía. También se incluyeron en este grupo pacientes con ECV con angioplastia, injertos, prótesis coronarias, marcapaso cardíaco y cambios vasculares con aplicación de prótesis.
De la lista oficial de 1947 enfermedades huérfanas reconocidas en el país, solo se encontró el $50 \%$ de los códigos CIE-10 (8). Debido a que muchas enfermedades huérfanas afectan otros sistemas, como el cardiovascular o el renal, se controló la duplicación de los registros. Entre las enfermedades neurológicas, se incluyeron tres categorías: epilepsia, estado de mal epiléptico, discapacidades y enfermedades crónicas incapacitantes por esta causa.

En trauma mayor que requiere unidad de cuidados intensivos se consideró el gran quemado (solo quemaduras de segundo y tercer grado, tanto por frío como por calor). Este grupo no incluyó otros eventos traumáticos, por indefinición de la causa. En enfermedades reumáticas se consideraron cinco condiciones clínicas, en concordancia con la Resolución 1393 del 2015 (20,21): lupus/artropatías, artritis reumatoides, artritis juvenil, poliartritis y otras artritis.

Con relación al VIH/sida, se identificaron seis subgrupos correspondientes a la codificación CIE-10: enfermedad por VIH resultante en enfermedades infecciosas y parasitarias, VIH resultante en tumores malignos, VIH resultante en otras enfermedades especificadas, VIH resultante en otras afecciones y VIH sin otra especificación/demencia.

Las variables sociodemográficas incluidas fueron edad y sexo. Las variables relacionadas con las características de la oferta incluyeron el tipo de servicio (consulta externa, hospitalización y urgencias), el régimen de afiliación, la naturaleza de la IPS (pública o privado), el profesional que atendió la consulta según la agrupación de la Clasificación Única de Procedimientos en Salud (CUPS), y, para los servicios de hospitalización y urgencias, se incluyó el estado de salida (vivo-muerto). 


\section{Análisis estadístico}

La información de la utilización de los servicios para cada EAC se describió con estadísticas descriptivas, según las variables sociodemográficas y las características de la oferta definidas arriba. Se realizaron análisis uni y bivariados, con sus respectivas medidas de tendencia. Se utilizaron frecuencias absolutas y relativas para las variables cualitativas y mediana con rango intercuartílico (RI) para las variables cuantitativas, dada la distribución heterogénea de los datos. Los datos fueron analizados con el paquete estadístico Statical Package for the Social Sciences (SPSS) versión 15.0. Los resultados se presentan en tablas.

\section{Consideraciones éticas}

El proyecto tuvo aprobación del Comité Técnico de Investigaciones y del Comité de Ética de la Investigación de la Facultad Nacional de Salud Pública, bajo la clasificación sin riesgo.

\section{Resultados}

La base de datos de los RIPS del 2011 contenía un total de 11121054 registros de utilización de servicios de salud por consulta externa, 241117 por hospitalización y 493758 por urgencias en el Departamento de Antioquia. De estos, 1869292 (15.7\%) correspondieron a pacientes con EAC. Entre los pacientes con EAC, 1820323 (97.4\%) fueron atendidos por consulta externa, 29794 (1.6\%) por hospitalización y 19175 (1\%) por urgencias.
Del total de atenciones de los pacientes con EAC, solo se consideró como evento catastrófico $9.5 \%$ de la utilización en los servicios de hospitalización y urgencias, pero no fueron clasificadas en el servicio de consulta externa, por falta de datos. En enfermedades como el VIH y el cáncer, la utilización de servicios de hospitalización por eventos específicamente considerados de alto costo supera el $20 \%$.

La ERC-P, el cáncer y la ECV fueron responsables del $82 \%$ de la utilización de los servicios (1527 927). Las enfermedades huérfanas ocuparon el segundo lugar de frecuencia de utilización total, correspondiente al $9.3 \%$ (174111), y la artritis el tercer lugar con 5.7\% (107209) de la utilización en todos los servicios.

Los patrones de consulta variaron entre los tres servicios. En consulta externa y urgencias, la mayor utilización se produjo por ERC-P (75.6 y $38.9 \%$, respectivamente), mientras que la utilización más frecuente en hospitalización fue por pacientes con cáncer, 11502 (38.6\%), seguida por ERC-P, $5534(18.6 \%)$. La utilización más frecuente en el servicio de urgencias por pacientes con EAC fue por ERC-P (39\%), seguida por el cáncer $(20 \%)$ y las enfermedades huérfanas (14.2\%) (véase tabla 1).

De los 28950 pacientes hospitalizados que tenían dato de estado de salida, el $2.8 \%$ (844) egresó muerto. La menor mortalidad al egreso se presentó entre los pacientes hospitalizados por artritis $(0.4 \%)$ y la mayor entre aquellos hospitalizados por $\mathrm{VIH}(4.8 \%)$. De las personas que ingresaron a urgencias, el $2.8 \%$ falleció, siendo mayor en los casos de VIH y cáncer. 


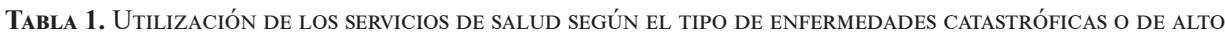
Costo. Antioquia, 2011

\begin{tabular}{|l|l|l|l|}
\hline \multirow{2}{*}{ Tipo de EAC } & \multicolumn{3}{|c|}{$\begin{array}{c}\text { Tipo de } \\
\text { servicio }\end{array}$} \\
\cline { 2 - 4 } & $\begin{array}{l}\text { Consulta, n: 1 820 323 } \\
\text { Frecuencia (\%) }\end{array}$ & $\begin{array}{l}\text { Hospitalización, n: 29 794 } \\
\text { Frecuencia (\%) }\end{array}$ & $\begin{array}{l}\text { Urgencias, n: 19 175 } \\
\text { Frecuencia (\%) }\end{array}$ \\
\hline ERC-P* & $1376650(75.6)$ & $553418.6)$ & $7464(38.9)$ \\
\hline Huérfanas & $168085(9.2)$ & $3298(11.1)$ & $2728(14.2)$ \\
\hline Artritis & $103068(5.7)$ & $3051(10.2)$ & $1090(5.7)$ \\
\hline Cáncer & $93889(5.2)$ & $11502(38.6)$ & $3764(19.6)$ \\
\hline Epilepsia & $31961(1.8)$ & $1078(3.6)$ & $1030(5.4)$ \\
\hline ECV & $23240(1.3)$ & $4561(15.3)$ & $1323(6.9)$ \\
\hline VIH & $18507(1.0)$ & $875(2.9)$ & $581(3.0)$ \\
\hline Gran quemado & $4923(0.3)$ & $306(1.0)$ & $1195(6.2)$ \\
\hline
\end{tabular}

ERC-P: constituye la ERC y las enfermedades precursoras

\section{Sexo}

En los tres servicios se observó una mayor frecuencia de utilización por el grupo de individuos $\geq 45$ años, que representó un promedio de utilización de $70 \%$. La utilización de los tres tipos de servicios se realizó predominante por mujeres $(62 \%)$, pero la proporción de consultas varió por servicio y enfermedad, siendo un poco más baja en urgencias $(54.3 \%)$. La utilización fue más frecuente en hombres para los tres servicios en el caso del VIH (84-87\%) y la ECV (52$55 \%)$. La brecha de utilización de consulta externa fue mayor en artritis, para la cual las mujeres absorbieron el $75.8 \%$, y en los hombres en el servicio de hospitalización por VIH, gran quemado y ECV (razón H:M de 5-1.6 y 1.2, respectivamente). En el caso de las urgencias, la utilización también fue más frecuente en mujeres, excepto para ECV, VIH, epilepsia y gran quemado.

\section{Edad}

La mediana de la edad en el servicio de consulta externa fue de cuarenta años (RI:
22-57), en el de hospitalización 38 (RI 21-61) y en urgencias de veintinueve (RI 17-46). La utilización de servicios de consulta para cada EAC sigue un patrón muy similar, aumentando a medida que aumenta la edad, excepto para VIH y gran quemado.

En menores de dieciocho años con EAC, se observó una utilización general de servicios de 51425/1869292 (2.8\%), aunque 9.1 y $9.2 \%$ de la utilización de los servicios de hospitalización y urgencias, respectivamente, se presentaron en esta franja de edad. La utilización de servicios de salud más frecuente en este grupo se produjo por enfermedades relacionadas con epilepsia, quemaduras y enfermedades huérfanas. La utilización promedio para la franja de población adulta, en edad productiva (entre 18 y 44 años) fue $21.8 \%$. Para los tres servicios, la frecuencia de utilización fue más alta por consultas relacionadas con VIH. El gran quemado, las enfermedades huérfanas y la epilepsia también fueron causas frecuentes de utilización en este grupo (tabla 2). 


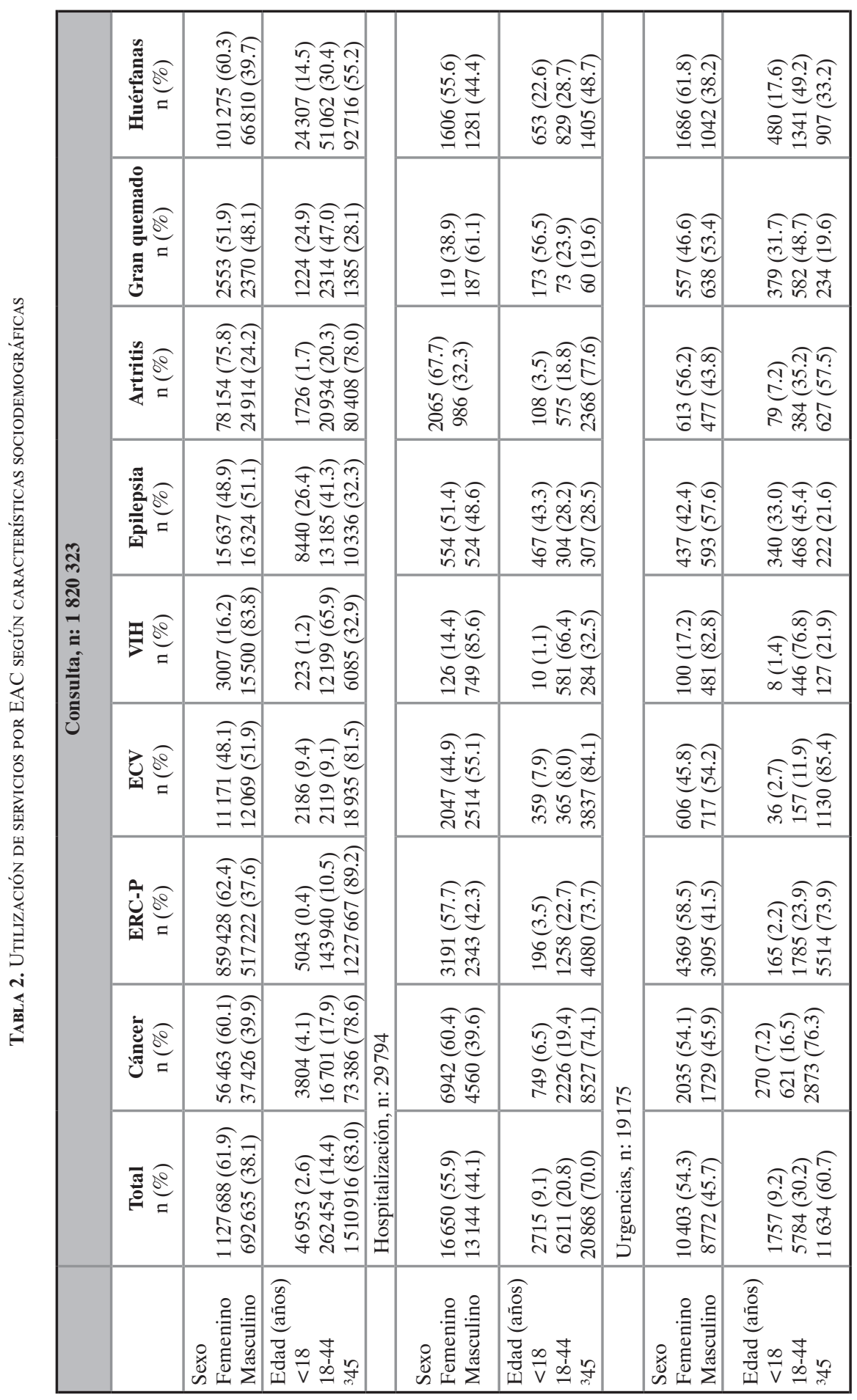

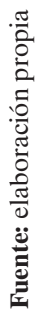




\section{Características de la oferta}

Régimen de afiliación al Sistema General de Seguridad Social en Salud. El 99.4\% de los registros de atención relacionados con la EAC fue reportado como consultas en población asegurada. Entre el 78 y el $82 \%$ de la utilización en los tres servicios fue registrado en población afiliada al régimen contributivo, y del total de las consultas realizadas en este último, el $0.4 \%$ se dirigió a la población de regímenes especiales. El $0.6 \%$ (11873) de la utilización se produjo en población pobre no asegurada; 10228 $(0.6 \%)$ eventos atendidos por consulta externa, $1123(3.8 \%)$ por hospitalización y
$522(2.7 \%)$ por urgencia. Solo el $0.1 \%$ de las consultas (2382) fue provisto por medicina prepagada. La proporción de hospitalizaciones realizadas en el régimen especial y en la población usuaria de la medicina prepagada fue marginal ( 0.5 y $1.7 \%$, respectivamente) (tabla 3).

El análisis por enfermedad mostró una razón de utilización régimen contributivo-régimen subsidiado de 5-9. Esta brecha en la utilización sobrepasó las nueve veces para enfermedades como: VIH, huérfanas, ECV y cáncer en consulta externa, artritis en el servicio de hospitalización y gran quemado en el servicio de urgencias (tabla 3).

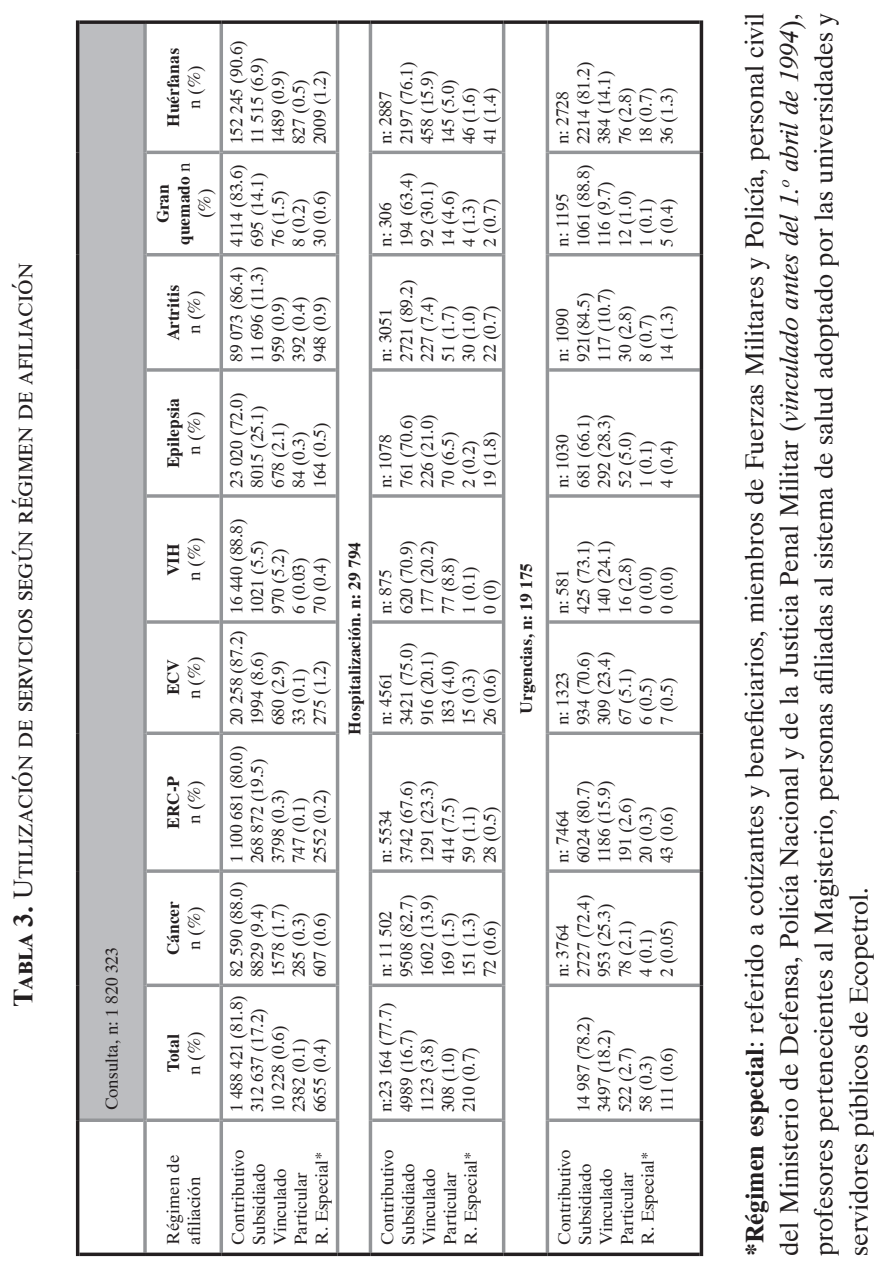


Naturaleza de la institución que atendió el servicio - Relación público- privado. Entre el 66 y el $82 \%$ de la utilización se produjo en IPS privadas, con una razón privada-pública de 4.5:1. Esta relación difirió de acuerdo con el tipo de enfermedad. En consulta externa, la mayor utilización de IPS privadas se produjo para la atención del cáncer, seguido por enfermedades huérfanas. La razón de atención privada-pública en servicios de hospitalización fue de 5.3. En este servicio la consulta privada predominó en los casos de ECV y artritis, con una razón de 12.1. En urgencias, la atención del paciente con artritis fue cinco veces mayor en IPS privadas que en las públicas (véase tabla 4).

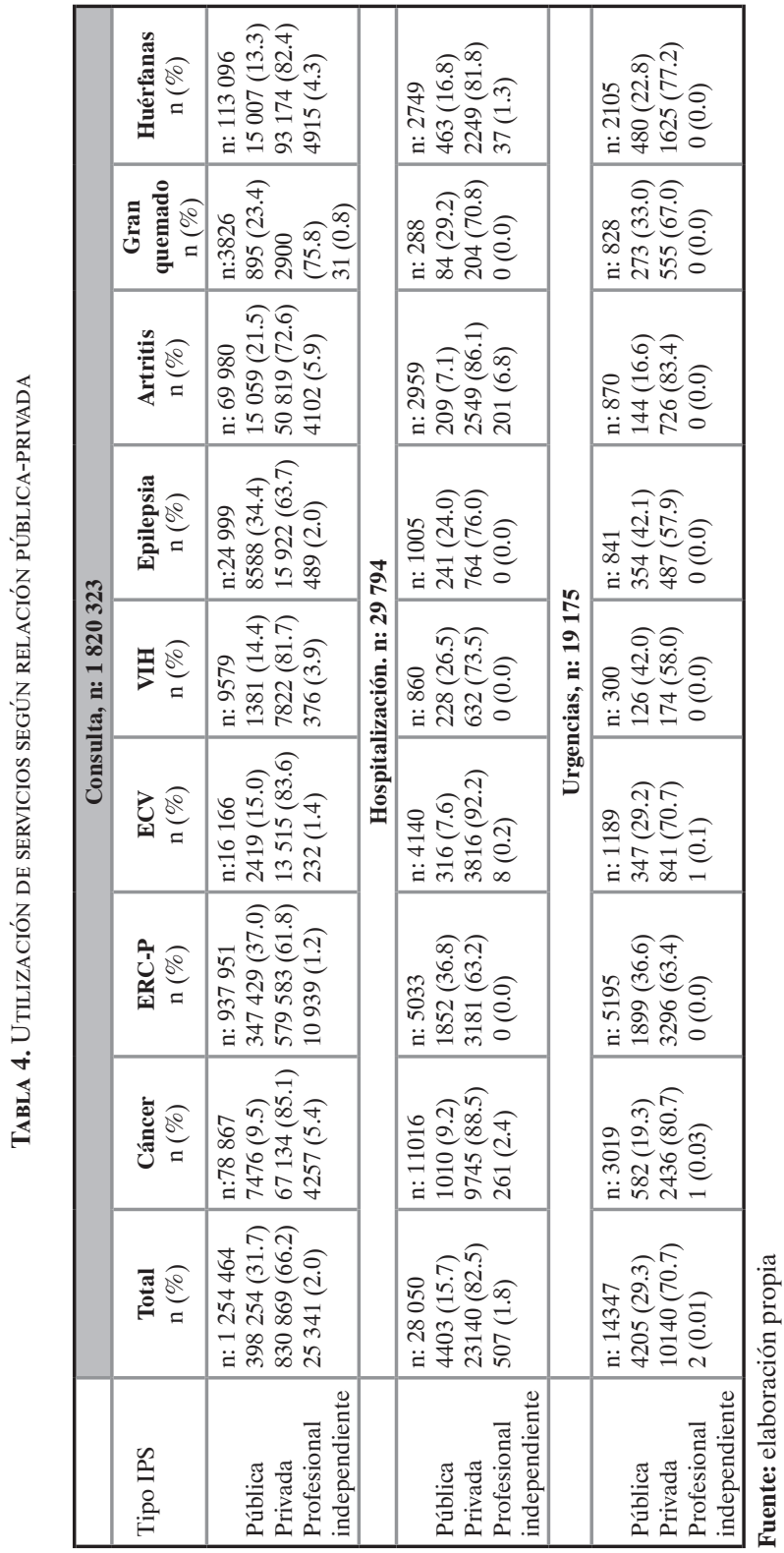


Tipo de profesional por enfermedad (consulta externa, hospitalización y urgencias) por enfermedades catastróficas o de alto costo. En la utilización de servicios de salud por consulta externa predominó la atención por medicina general para todas las enfermedades, excepto en los casos de cáncer. Por cada consulta médica especializada se realizaron entre una y nueve consultas con médico general, siendo las más frecuentes aquellas generadas para la atención de eventos relacionados con la ERC-P (nueve veces) y la atención por quemados (siete veces). La relación es invertida en el caso del cáncer, donde por cada consulta con médico general se realizan 2.4 consultas por especialista.

La utilización del servicio de consulta externa realizada por equipo interdisciplinario y especialidades diferentes a la médica fue inferior al $5 \%$, excepto para el cáncer, la ECV y el VIH, cuya atención por equipo interdisciplinario se realizó entre el 10 y el $24 \%$ de los casos atendidos. El número de consultas de nutrición fue inferior al $5 \%$, excepto para las enfermedades huérfanas, caso en el cual fue ligeramente superior.

\section{Discusión}

En este estudio se encontró que la utilización de servicios de salud por parte de pacientes con enfermedades de alto costo varía de acuerdo con la enfermedad y los servicios, así como con el sexo, la edad y el régimen de afiliación. Por el contrario, para todas las enfermedades y en los tres servicios, la constante fue la utilización de servicios con predominio de IPS privadas. La provisión de los servicios se produjo principalmente por médicos generales, mientras que otros servicios, considerados parte de la atención integral en estas patologías tan complejas, se produjeron de forma marginal.
La utilización de servicios de salud fue mayor en mujeres que en hombres para todos los servicios, excepto en enfermedades como la ECV, el VIH, la epilepsia y el gran quemado, en las cuales la relación fue invertida.

La mayor utilización de los servicios de salud por mujeres ha sido reportada por múltiples autores alrededor del mundo, y se explica de forma multifactorial por las diferencias biológicas, un mayor reconocimiento de los síntomas, mejor percepción de la enfermedad y patrones de socialización estrechamente ligados a determinantes culturales, entre otros. Se ha afirmado también que las mujeres tienden a efectuar una mayor búsqueda de servicios preventivos, lo que aumenta la probabilidad de recibir diagnósticos y tratamientos que impliquen la necesidad de más servicios $(1,22)$. La mayor utilización de servicios de salud entre los hombres para ECV y VIH probablemente refleje el comportamiento epidemiológico de dichas enfermedades (23-25). Otros autores han sugerido que la menor utilización por hombres pudiera estar relacionada con fallas por parte de la oferta (22).

Un análisis sobre la mayor utilización de servicios de salud por mujeres implicaría conocer muy bien la epidemiología de cada enfermedad y establecer, de ese modo, si estas diferencias halladas son producto de procesos biológicos o, por el contrario, son el resultado de procesos evitables e injustos, aspectos de los cuales este estudio no puede dar cuenta. Por otra parte, la información disponible no discrimina la gravedad de la situación que generó la utilización, por lo tanto, no es posible hacer un análisis de género que permita comprender este fenómeno.

Con relación a la utilización por edad, las personas mayores de cincuenta años absorbieron entre el 60 y el $72 \%$ de la utilización en los tres tipos de servicios, sin embargo, una 
cuarta parte de la utilización de los servicios de urgencias y hospitalización se produjo en población de adultos jóvenes y un $7 \%$ en menores de dieciocho años. La mayor utilización de servicios en adultos mayores ha sido reportada en otros trabajos alrededor del mundo y es un fenómeno comprensible, puesto que muchas de estas enfermedades crónicas, sus comorbilidades y enfermedades discapacidades se presentan con mayor frecuencia en esta población (26-28). Por el contrario, la utilización de servicios de salud en servicios de urgencia y hospitalización por adultos jóvenes en este grupo de enfermedades es un fenómeno inesperado, cuya comprensión amerita nuevos estudios. Algunos estudios han informado que la utilización de servicios de urgencias se produce en ausencia de mecanismos eficaces para el acceso a servicios de consulta externa, por motivaciones personales o porque no quiere o no puede asistir a la consulta regular (29). El acceso a los servicios de salud por urgencias implica mayores costos para el sistema de salud, y debería ser evitado, mediante el acceso efectivo y con continuidad de los pacientes con EAC a programas especiales que reduzcan la velocidad de progresión.

Algunos autores explican los diferenciales en la utilización de servicios por factores estructurales e institucionales como la organización del sistema de salud y sus esquemas de financiamiento, las diferencias inherentes al tipo de modelo asistencial adoptado y el tipo de aseguramiento $(30,31)$. En este estudio se encontró que la utilización de servicios por EAC fue mayor en el régimen contributivo que en el subsidiado, lo cual contrasta con el número de personas afiliadas en ese año a este régimen en el departamento de Antioquia (53.4\%) (32).

Por otra parte, para la fecha, un porcentaje bajo pero importante $(0.6 \%)$ de la consulta externa, el $3.8 \%$ de la hospitalización y el $2.7 \%$ de las urgencias, se produjo en población pobre no asegurada (PNA). Por norma, la atención de la PNA que padece alguna EAC debe estar cubierta por recursos del Fondo de Solidaridad y Garantía y del fondo de enfermedades catastróficas. De manera que el departamento de Antioquia debió asumir por este concepto importantes gastos injustificables, en razón de la ética y la eficiencia económica. Estos gastos resultan inaceptables, toda vez que se evitarían al afiliar al total de la población y pagar los valores correspondientes a la UPC anual (determinada para ese año en \$30204000 de pesos, según el Acuerdo 219 de la Comisión de Regulación en Salud (CRES) (33), y resulta especialmente importante para el caso de las hospitalizaciones y las urgencias.

La baja utilización de servicios de salud en personas del régimen subsidiado y PNA, en comparación con la utilización de servicios por personas del régimen contributivo, no tiene una justificación epidemiológica, lo que sugiere inequidades en la utilización de servicios relacionadas con la seguridad social bajo un modelo segmentado.

Es conocido que la población del régimen subsidiado y la población PNA están conformadas por una gran proporción de población campesina y minorías étnicas, población residente de zonas dispersas, de difícil acceso geográfico a los servicios de salud, difícil desplazamiento a las grandes ciudades, que son las que concentran el servicio de tercer y cuarto nivel de atención. En el grupo afiliado al régimen subsidiado y al PNA se encuentran personas en vulnerabilidad respecto a su condición laboral y financiera y también aquellas que carecen de acceso debido a las grandes dificultades en que se encuentran los hospitales públicos del país (34). La inequidad en la utilización no es un fenómeno exclusivo en Colombia, pero sí predomina en sistemas de salud fragmentados. Por el 
contrario, algunos estudios han mostrado que en países con sistemas de protección social fuerte, como Reino Unido, Canadá o Dinamarca, la utilización es mayor en poblaciones con más desventajas (1).

La inequidad en salud no se expresa solamente en diferenciales en la atención de acuerdo con las características socioeconómicas de la población, sino que también se relaciona con la asignación de los recursos y con la organización de la red de servicios. Con respecto a este último punto, nuestro análisis permitió concluir que la red de servicios para la atención de este tipo de enfermedades fue predominantemente privada. $\mathrm{La}$ baja relación en la utilización pública-privada pudiera ser explicada por las políticas de privatización que de manera progresiva han llevado a la desfinanciación y crisis del sector público relacionada con los mecanismos de contratación y pago de los servicios, así como con los costos de operación (35-39) La menor utilización de servicios por EAC de la población del régimen subsidiado y los PNA encontrada en este estudio coincide con la menor utilización en las IPS públicas.

Un aspecto importante que destacar es la escasa participación de la medicina prepagada en la atención de las EAC, lo cual sugiere que a pesar de las altas tarifas cargadas a los usuarios, la carga financiera para la atención de estas complejas enfermedades se traslada al SGSSS, y, por tanto, se estima un alto margen de ganancia en desmedro de las finanzas del sector salud.

Con relación propiamente a las características en la prestación del servicio, la fuente no discriminó las consultas referentes a prevención de la enfermedad, ni la utilización de servicios con propósitos de rehabilitación. Por lo tanto, no fue posible efectuar este tipo de análisis. Sin embargo, se sabe por datos recientes de la Encuesta de Calidad de Vida (ENCV) que el $27 \%$ de las personas no hacen consultas de prevención de la enfermedad (40).

Por otra parte, sí es posible establecer que, aun cuando se trata de enfermedades clínicamente complejas y progresivas, que ameritan manejo integral, el servicio en consulta externa fue provisto mayoritariamente por personal médico general, con utilización marginal de servicios como nutrición, psicología y trabajo social (inferior al $5 \%$ ). La complejidad clínica de estas enfermedades, las comorbilidades asociadas, su cronicidad y la necesidad de una adecuada adherencia al tratamiento exigen una atención integral $(41,42)$. Las guías clínicas nacionales de manejo y una amplia bibliografía internacional así lo han justificado (8,19 42-53).

Este asunto resulta crítico, toda vez que, por ejemplo, los pacientes con ERC sufren mayor prevalencia de malnutrición calórica-proteica (54) y los pacientes con VIH registran cifras alteradas de metabolitos que son indicadores bioquímicos del estado nutricional (55). En el país se ha evidenciado un porcentaje de desnutrición y de malnutrición que puede llegar al $64 \%$ en pacientes pediátricos con VIH (56).

En el caso de los pacientes quemados, es conocido que los trastornos nutricionales y emocionales interfieren en la respuesta al tratamiento médico-quirúrgico. Estos trastornos aumentan las tasas de eventos adversos y, en consecuencia, la estancia hospitalaria y los costos de atención médica (57). Una situación similar sucede en los pacientes críticos sometidos a hospitalización, en quienes se han encontrado tasas de malnutrición cercanas a 30-60\%. Esta malnutrición puede ser preexistente o exacerbarse por el estado hipercatabólico e hipermetabólico (58). El control dietario en pacientes afectados con EAC es parte del plan de tratamiento y se 
justifica para mantener el balance metabólico, evitar el deterioro de la condición de vida, retrasar la progresión de la enfermedad a estadios más avanzados, así como mejorar la eficacia del tratamiento farmacológico. Simultáneamente con el manejo nutricional, se han señalado otras necesidades de atención psicosocial para pacientes con enfermedades crónicas no transmisibles (59), como ECV (60) y pacientes quemados (61), entre otros.

En cuanto a los egresos de hospitalización y urgencias, nuestro estudio mostró que $2.8 \%$ de los pacientes con EAC, quienes utilizan estos servicios, egresan muertos. Esta cifra está muy por encima de aquellas reportadas en Medellín ( $0.2 \%)$, donde se consideró la utilización por todas las causas (29), y puede indicar que los pacientes con EAC llegan descompensados o en condiciones críticas de salud a estos servicios. Se sugiere desarrollar otros estudios que permitan entender cómo se produce el contínuum de servicios y programas para el manejo de esta población y qué relación tiene con la alta mortalidad, cuando son enfermedades las más de las veces prevenibles.

Solo el $9.5 \%$ de la utilización de servicios de hospitalización y urgencias se reportó como eventos de alto costo. La definición restringida de las enfermedades y los tratamientos de alto costo impone varios retos y contradicciones para la utilización de los servicios. El primero de ellos relacionado con la enfermedad misma, puesto que el manejo de los pacientes con EAC se hace por evento, y no como parte de un programa especial de manejo. Este tipo de manejo no permite efectuar monitoreo y seguimiento al paciente y puede acarrear consecuencias adversas desde el punto de vista de la seguridad clínica. El segundo reto es que las 134 enfermedades priorizadas y los tratamientos considerados de alto costo están exentos de copagos y cuotas moderadoras (62), de tal manera que los pacientes con EAC que no cumplen con estas especificaciones pueden ser sometidos a barreras financieras que limitan la utilización de los servicios de salud.

El estudio mostró las características en la utilización de servicios que se concretó, sin embargo, se desconoce cuál es la demanda real de servicios relacionados con EAC. Un estudio de la Organización Panamericana de la Salud (OPS), llevado a cabo en el 2002, identificó que solo el $68.5 \%$ de los hombres y el $70.9 \%$ de las mujeres enfermas utilizan el servicio de salud (22). Otro estudio en la ciudad de Medellín indicó que el 10.6\% de los pacientes que buscaron atención por el servicio de urgencia no fueron atendidos (29).

En este estudio tampoco fue posible evidenciar otros atributos de calidad en la prestación de servicios, referidos a la captación, continuidad o la oportunidad, frecuencia de uso, progresión de la enfermedad o reingresos. Esta información es necesaria para entender la situación de las EAC y la respuesta del sistema de salud. Sería deseable que estos datos fueran recolectados por los agentes prestadores. Alternativamente, se recomienda abordar estos aspectos en nuevas investigaciones. Estos vacíos de conocimiento deben ser suplidos en futuras investigaciones.

\section{Limitaciones del estudio}

Las variables utilizadas en la base de datos de los RIPS son reportadas por las distintas IPS, por lo tanto, su fiabilidad está sujeta a la veracidad del reporte. Es bien sabido que los RIPS presentan problemas de calidad del dato, información no desagregada y ausencia de otras variables sociodemográficas que permitan hacer análisis más exhaustivos. Con la calidad actual de las bases de datos, no es posible estimar la frecuencia de uso, y, por lo tanto, se desconoce el porcentaje atribuible 
al uso excesivo de los servicios de salud. Este fenómeno es susceptible de analizarse en futuras investigaciones.

Como la información no está discriminada de acuerdo con la zona geográfica, urbana-rural, tampoco es posible identificar los patrones o brechas de utilización en los distintos territorios.

En Colombia, las enfermedades huérfanas están definidas como aquellas raras, ultrahuérfanas u olvidadas y conforman una lista de 1947 entidades. En el país, de esta lista solo está codificado el $50 \%$, según la Clasificación Internacional de Enfermedades (CIE-10). Por consiguiente, se estima que la situación de la utilización para este tipo de enfermedad puede ser mayor que la reportada aquí. Mientras no se homologuen los códigos con el sistema internacional sugerido por Orphanet, no será posible tener datos epidemiológicos o administrativos certeros para este grupo de enfermedades.

A pesar de las limitaciones anteriores, esta es la única fuente oficial de base poblacional que permite identificar la utilización de los servicios y, en consecuencia, después del esfuerzo y los costos que implican el registro y la recolección de los datos allí contenidos, no sería ético descartarla. Se sugiere que la autoridad sanitaria implemente procesos para mejorar los sistemas de información y vigilancia en salud pública que contemplen variables útiles para el monitoreo de la calidad de dicha utilización.

\section{Agradecimientos}

Al magíster en Estadística Eduardo Hurtado y a la magíster en Epidemiología Libia María Rodríguez Padilla, por su apoyo en el análisis y la presentación de los datos. A Erika Ríos, miembro del Semillero de Investigación, por su apoyo en la búsqueda de información. Al Fondo de Apoyo Docente, por la financiación de este estudio.

\section{Referencias bibliográficas}

1. Mendoza-Sassi R, Béria J. Utilización de los servicios de salud: una revisión sistemática sobre los factores relacionados. Cad Saúde Pública. 2001;17(4):819-32.

2. Arredondo A, Meléndez V. Modelos explicativos sobre la utilización de servicios de salud: revisión y análisis. Salud Pública de México. 1992;34(1):36-49.

3. Faulkner D, Law J. The 'unnecessary' use of emergency departments by older people: findings from hospital data, hospital staff and older people. Aust Health Rev. 2015;39(15):544-51.

4. Guimarães DS, Soares EJO, Júnior GF, Medeiros DD. Attributes and circumstances that induce inappropriate health services demand: a study of the health sector in Brazil. BMC Health Services Research. 2015;15(65):1-8.

5. Segal JB, Nassery N, Chang H-Y, Chang E, Chan $\mathrm{K}$, Bridges JF. An index for measuring overuse of health care resources with Medicare claims. Med Care. 2015;53(3):230-6.

6. Colombia, Ministerio de Salud y Protección Social. Análisis de Situación de Salud (ASIS). Bogotá D. C.: Dirección de Epidemiología y Demografía; 2015.

7. Colombia, Ministerio de Salud. Resolución 5261. Por la cual se establece el Manual de Actividades, Intervenciones y Procedimientos del Plan Obligatorio de Salud en el Sistema General de Seguridad Social en Salud. Bogotá: Ministerio de Salud y Protección Social; 1994.

8. Colombia, Ministerio de Salud y Protección Social. Decreto 430. Por el cual se define el listado de las enfermedades huérfanas. Bogotá: Ministerio de Salud y Protección Social 2013.

9. Cuenta de Alto Costo. Enfermedades huérfanas. Bogotá: Cuenta de Alto Costo; 2012 [acceso: 12 de mayo del 2016]. Disponible en: http://www. cuentadealtocosto.org/site/index.php/9-patologias/24-enfermedades-huerfanas/?template $=$ cuentadealtocostocontenido.

10. Organización Mundial de la Salud (OMS). Informe sobre la situación mundial de las enfermedades no transmisibles Ginebra: OMS; 2014 [acceso: 22 de noviembre del 2016]. Disponible en: http:// apps.who.int/iris/bitstream/10665/149296/1/WHO NMH_NVI_15.1_spa.pdf?ua $=1 \& u a=1$

11. Organización Mundial de la Salud (OMS). Diabetes Ginebra 2016 [acceso: 24 de noviembre del 2016]. Disponible en: http://www.who.int/mediacentre/factsheets/fs312/es/

12. Cuenta de Alto Costo. Situación de la enfermedad renal crónica, hipertensión arterial y diabetes mellitus. Bogotá D.C.: Cuenta de Alto Costo; 2015.

13. Colombia, Ministerio de Salud, Instituto Nacional de Salud. Mortalidad evitable en Colombia para 
1998-2011. Bogotá D. C.: Observatorio Nacional de Salud; 2014.

14. Carrizosa Moog J. Prevalencia, incidencia y brecha terapéutica en la epilepsia. Iatreia. 2007;20(3):28296.

15. Pradilla G, Vesga BE, León-Sarmiento FE. Estudio neuroepidemiológico nacional (Epineuro) colombiano. Revista Panamericana de Salud Pública. 2003;14(2):104-11.

16. Colombia, Ministerio de Salud y Protección Social, Instituto Nacional de Salud. Protocolo de vigilancia en salud pública enfermedades huérfanas-raras. Bogotá: Federación Colombiana de Enfermedades Raras; 2016.

17. Lizcano D, Palacios A, Restrepo J, González A. Enfermedades crónicas no transmisibles. Estructura de la morbilidad en el departamento de Antioquia. Medellín: Gobernación de Antioquia; 2012.

18. Santacruz P, Dorta L. La carga de la enfermedad renal crónica (ERC) sobre la mortalidad de la población. ¿Los datos disponibles reflejan la realidad? Nefrología. 2006;26(4):419-20.

19. National-Kidney-Foundation. KDOQI clinical practice guideline for hemodialysis adequacy: 2015 Update. U.S: The National Kidney Foundation Kidney Disease Outcomes Quality Initiative (NKF KDOQI); 2015 [acceso: 13 de abril del 2016]. Disponible en: http://www.ajkd.org/article/S02726386(15)01019-7/pdf

20. Colombia, Ministerio de Salud y Protección Social. Resolución 1393 de 2015. Por la cual se establece el registro de pacientes con artritis reumatoide a la Cuenta de Alto Costo. Bogotá D. C.: Ministerio de Salud; 2015.

21. Caballero Uribe CV. Artritis reumatoide como enfermedad de alto costo. Revista Colombiana de Reumatología. 2002;11(3):225-31.

22. Gómez Gómez E. Género, equidad y acceso a los servicios de salud: una aproximación empírica. Rev Panam Salud Publica. 2002;11(5/6):327-34.

23. Gómez J. Morbimortalidad cardiovascular en el mundo. Revista Colombiana de Cardiología. 2012;19(6):298-9.

24. Osorio E, Luque R, Ávila S. Boletín Epidemiológico, situación del VIH/sida, Colombia. Ministerio de Salud: Bogotá, D. C.; 2013 [acceso: 16 de septiembre del 2016]. Disponible en: https://www. minsalud.gov.co/Documentos $\% 20 y \% 20$ Publicaciones/BOLETIN\%20EPIDEMIOLOGICO\%20 VIH\%201983-2012.pdf

25. Colombia, Instituto Nacional de Salud. Enfermedad cardiovascular: principal causa de muerte en Colombia. Observatorio Nacional de Salud. 2013; Boletín(1):1-6.

26. Wong R, Peláez M, Palloni A. Autoinforme de salud general en adultos mayores de América Latina y el Caribe: su utilidad como indicador. Rev Panam Salud Publica. 2005;17(5/6):323-32.

27. Llibre Guerra JC, Guerra Hernández MA, Perera Miniet E. Comportamiento de las enfermedades crónicas no transmisibles en adultos mayores. Revista Cubana de Medicina General Integral. 2008;24.
28. Organización Mundial de la Salud (OMS). Informe sobre la situación mundial de las enfermedades no transmisibles 2014. Cumplimiento de las nueve metas mundiales relativas a las enfermedades no transmisibles: una responsabilidad compartida. 2014 [acceso: 12 de abril del 2016]. Disponible en: http://apps.who.int/iris/bitstream/10665/149296/1/ WHO_NMH_NVI_15.1_spa.pdf?ua $=1$

29. Valencia-Sierra $\bar{M} L$, González-Echeverri G, Agudelo-Vanegas NA, Acevedo-Arenas L, Vallejo-Zapata IC. Acceso a los servicios de urgencias en Medellín, 2006. Revista de Salud Pública. 2007;9(4):529-40.

30. Llanos AA, Morera-Salas M, Barber-Pérez P, Hernández K, Xirinach-Salazar Y, Varga JR. Factores relacionados con la utilización de servicios de salud en Costa Rica. Revista de Salud Pública. 2009;11:323-35.

31. Oliveira G, Silva S. Encuesta sobre la utilización de los servicios de salud por hombres adultos: prevalencias y factores asociados. Rev Latino-Am Enfermagem [Internet]. 2016;(24):1-9.

32. Colombia, Departamento Administrativo Nacional de Estadística. Encuesta Nacional de Calidad de Vida 2011. Bogotá: DANE; 2011 [acceso: 25 de mayo del 2016]. Disponible en: http://www.dane. gov.co/files/investigaciones/condiciones_vida/calidad_vida/ECV_2011_Antioquia.pdf

33. Colombia, Comisión de Regulación en Salud. Acuerdo N. ${ }^{\circ} 219$. Por el cual se fija el valor de la Unidad de Pago por Capitación del Plan Obligatorio de Salud de los Regímenes Contributivo y Subsidiado para el año 2011. Diario Oficial 47939 de diciembre 31. 2010.

34. Nieto-Enciso LH. Análisis del comportamiento de la siniestralidad por enfermedades catastróficas en una empresa promotora de salud-Colombia. Rev Salud Pública. 2005;7(3):293-304.

35. Congreso de la República de Colombia. Ley 100. Por la cual se crea el sistema de seguridad social integral y se dictan otras disposiciones. 1993 [acceso: 26 de mayo del 2016]. Disponible en: http:// www.alcaldiabogota.gov.co/sisjur/normas/Norma1. jsp? $\mathrm{i}=5248$

36. República de Colombia. Decreto 4747. Por medio del cual se regulan algunos aspectos de las relaciones entre los prestadores de servicios de salud y las entidades responsables del pago de los servicios de salud de la población a su cargo, y se dictan otras disposiciones. Bogotá: Ministerio de la Protección Social; 2007 [acceso: 26 de mayo del 2016]. Disponible en: http://www.ins.gov.co:81/ normatividad/Decretos/DECRETO $\% 204747 \% 20$ DE\%202007.pdf

37. Gómez LA. La crisis hospitalaria: análisis de la producción y el pago por servicios individuales de salud en un hospital de primer nivel de atención de la red Pública de Bogotá. Revista Salud Bosque. 2012;2(2):61-8.

38. Congreso de la República de Colombia. Ley 1438. Por medio de la cual se reforma el Sistema General de Seguridad Social en Salud y se dictan 
otras disposiciones. Bogotá, D. C.: Diario Oficial N. ${ }^{\circ} 47.957 ; 2011$.

39. Asociación Colombiana de Hospitales y Clínicas. 27..$^{\circ}$ informe de seguimiento de cartera hospitalaria con corte a diciembre 31 de 2011. Bogotá: ACHC; 2012 [acceso: 26 de mayo del 2016]. Disponible en: http://achc.org.co/documentos/investigacion/estudios/achc/periodicos/cartera/27.INFORME_CARTERA_A_DICIEMBRE27_2011_(26_04_2012). pdf

40. Colombia, Departamento Administrativo Nacional de Estadística. Encuesta Nacional de Calidad de Vida 2015. Bogotá: DANE; 2016 [acceso: 25 de mayo del 2016]. Disponible en: http://www.dane. gov.co/files/investigaciones/condiciones_vida/calidad_vida/Presentacion_ECV_2015.pdf

41. Rincón-Hoyos HG, Castillo A, Reyes C, Toro CE, Rivas JC, Pérez Á, et al. Atención psicosocial integral en enfermedad catastrófica (Atinar). Revista Colombiana de Psiquiatría. 2006;35:44-71.

42. Saad Acosta C, Sepúlveda G, Ibáñez E, Flórez C, Herrán M, Márquez N, et al. Adherencia de los pacientes con insuficiencia renal crónica a las sesiones de hemodiálisis del Hospital Militar Central, Bogotá, D.C., 20062007 / Adhesion of hemodialysis sessions in patients with chronic kidney failure from Hospital Militar Central: Bogotá, D.C., 2006-2007. Revista Colombiana de Enfermería. 2008;3(3):61-7.

43. Colombia, Ministerio de Salud y Protección Social. Guía de práctica clínica (GPC) para el manejo del cáncer de cuello uterino invasivo. Bogotá: Instituto Nacional de Cancerología-ESE; 2014.

44. Colombia, Ministerio de Salud y Protección Social, Departamento Administrativo de Ciencia, Tecnología e Innovación en Salud. Guía de Práctica Clínica (GPC) para la detección temprana, tratamiento integral, seguimiento y rehabilitación del cáncer de mama. Bogotá: Ministerio de Salud; 2013.

45. Colombia, Ministerio de Salud y Protección Social, Departamento Administrativo de Ciencia Tecnología e Innovación en Salud. Guía de práctica clínica (GPC) para la detección temprana, diagnóstico, tratamiento, seguimiento y rehabilitación del cáncer de próstata. Bogotá: Cuenta de Alto Costo; 2013.

46. Colombia, Ministerio de Salud y Protección Social, Departamento Administrativo de Ciencia, Tecnología e Innovación en Salud. Guía de práctica clínica (GPC) para la detección temprana, diagnóstico, estadificación y tratamiento del cáncer de pulmón. Bogotá: Instituto Nacional de Cancerología; 2014.

47. Sociedad Colombiana de Cardiología y Cirugía Cardiovascular. Guías de prevención primaria de riesgo cardiovascular "tópicos selectos". Revista Colombiana de Cardiología. 2009;16(3).

48. Colombia, Ministerio de la Protección Social, Colciencias. Guía de práctica clínica. Hipertensión arterial temprana (HTA). Bogotá: Centro Nacional de Investigación en Evidencia y Tecnologías en Salud (CINETS); 2013.

49. Colombia, Ministerio de Salud y Protección Social, Fondo de Población de las Naciones Unidas. Guía de práctica clínica (GPC) basada en la evidencia científica para la atención de la infección por VIH/ sida en adolescentes (con 13 años de edad o más) y adultos. Bogotá: Asociación Colombiana de Infectología; 2014.

50. Colombia, Ministerio de Salud y Protección Social, Organización Panamericana de la Salud. Guía de práctica clínica (GPC) sobre diagnóstico y tratamiento de epilepsia. Bogotá: Instituto de Evaluación Tecnológica en Salud; 2014.

51. Colombiana de Salud S. A. Guía de manejo consulta especializada de reumatología. Artritis reumatoidea. Bogotá: Colombiana de Salud S. A.; 2012.

52. Colombiana de Salud S. A. Guía de identificación de pacientes con enfermedad renal crónica: 2015 2020. 2015 [acceso: 13 de abril del 2016]. Disponible en: http://www.colombianadesalud.org.co/MEDICINA/Guia\%20estadio\%20falla\%20renal.pdf

53. Colombia, Ministerio de la Protección Social, Naciones Unidas, Onusida. Manual de alimentación y nutrición para el cuidado y apoyo de personas adultas viviendo con VIH o con sida. 2010 [acceso: 25 de mayo del 2016]. Disponible en: https://www. minsalud.gov.co/salud/Documents/observatorio_ vih/documentos/atencion_integral/a.adultos/Manual\%20VIH\%20Nutricion\%20ADULTOS.pdf

54. De Luis D, Bustamante J. Aspectos nutricionales en la insuficiencia renal. Nefrología. 2008;28(3):33948.

55. Linares M, Bencomo J, Pérez L, Torres O, Barrera O. Influencia de la infección por VIH/sida sobre algunos indicadores bioquímicos del estado nutricional. Biomédica. 2002;22(2):116-22.

56. Ochoa V, Olaya M, Velasco C, López P. Malnutrición en niños colombianos con infección por VIH/ sida. Revista Gastrohnup. 2012;14(2):44-8.

57. Miquet Romero L, Rodríguez Garcell R, Barreto Penié J, Santana Porbén S. Estado de la provisión de cuidados nutricionales al paciente quemado: auditoría de procesos en un servicio de quemados de un hospital terciario. Nutrición Hospitalaria. 2008;23(4):354-65.

58. Montejo González JC, Culebras-Fernández JM, García de Lorenzo y Mateos A. Recomendaciones para la valoración nutricional del paciente crítico. Revista Médica de Chile. 2006;134(8):1049-56.

59. Orozco-Gómez Á, Castiblanco-Orozco L. Factores psicosociales e intervención psicológica en enfermedades crónicas no transmisibles Revista Colombiana de Psicología. 2015;24(1):203-17.

60. Rodríguez T. Manejo y orientación psicológica en el paciente con enfermedad cardiovascular en estado de gravedad. Revista Psicología Científica. 2011;13(1).

61. Gonçalves N, Echevarría-Guanilo ME, Carvalho FL, Inocenti Miasso A, Rossi LA. Factores biopsicosociales que interfieren en la rehabilitación de víctimas de quemaduras: revisión integradora de la literatura. Rev Latino-Am Enfermagem. 2011;19(3):622-30.

62. Consejo Nacional de Seguridad Social en Salud. Acuerdo 260. Por el cual se define el régimen de pagos compartidos y cuotas moderadoras dentro del Sistema General de Seguridad Social en Salud. Bogotá, D. C.: Diario Oficial 45474; 2004. 